\title{
Wearable Automatic Feedback Devices for Physical Activities
}

\author{
Daniel Spelmezan \\ RWTH Aachen University \\ 52056 Aachen, Germany \\ spelmezan@cs.rwth- \\ aachen.de
}

\author{
Adalbert Schanowski \\ RWTH Aachen University \\ 52056 Aachen, Germany \\ adalbert.schanowski@rwth- \\ aachen.de
}

\author{
Jan Borchers \\ RWTH Aachen University \\ 52056 Aachen, Germany \\ borchers@cs.rwth- \\ aachen.de
}

\begin{abstract}
With this work we want to illustrate new ways to assist students during sports training and to enhance their learning experience. As an example, we present the design of a wearable system intended for snowboard training on the slope. The hardware platform consists of a custom-built sensor/actuator box and a mobile phone acting as host device. These devices run algorithms for activity, context, and mistake recognition, and trigger feedback in response to classification results. Instructors can use such a system to automatically supervise posture and motion of students, i.e., to detect common mistakes that are difficult to recognize when observing students from far away, and to provide immediate audible or tactile feedback for corrections during courses. The presented approach can further be applied to supervise posture and to alert users to potentially harmful body movements performed during daily physical activities.
\end{abstract}

\section{Keywords}

HCI, mobile computing, wearable computing, assistive technologies, context awareness, tactile feedback, snowboarding.

\section{INTRODUCTION}

Learning new sports is often difficult and time consuming. Students need to practice for a long time until they can correctly perform the necessary techniques of a sports domain. In some sports, such as tennis or golf, instructors can always talk to students and explain correct technique. Moreover, an instructor can physically guide, for example, the student's arm to demonstrate correct strokes. Such frequent concurrent feedback during exercises can be beneficial for acquiring new motor skills [13]. In sports such as snowboarding or skiing, however, the instructor cannot be with the students while going down the slope. Due to this spatial separation, a snowboarding instructor cannot directly talk to students when they incorrectly perform exercises (Fig. 1). The instructor typically provides feedback after exercises.

Permission to make digital or hard copies of all or part of this work for personal or classroom use is granted without fee provided that copies are not made or distributed for profit or commercial advantage and that copies bear this notice and the full citation on the first page. To copy otherwise, to republish, to post on servers or to redistribute to lists, requires prior specific permission and/or a fee.

BodyNets '09 Los Angeles, CA, USA

Copyright 2008 ICST 978-963-9799-41-7 ...\$5.00.

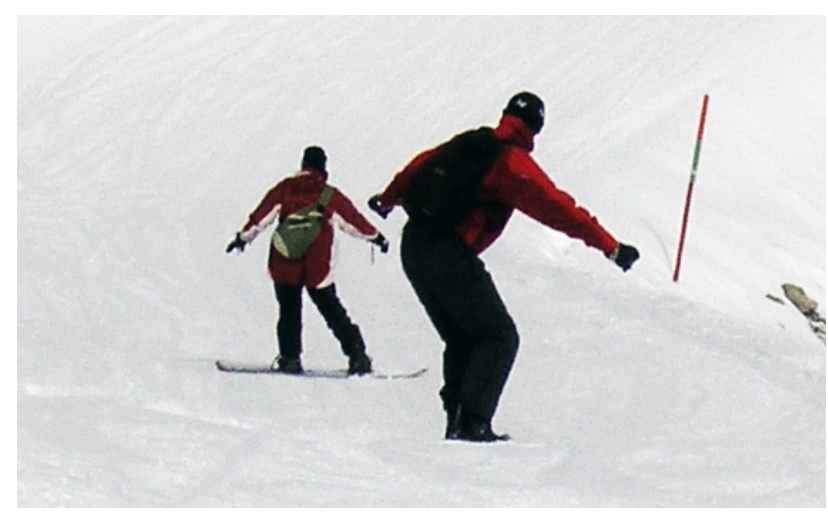

Figure 1: Two beginners descend the slope. The instructor cannot talk to the students to give them feedback on their mistakes.

We present our work on wearable automatic feedback devices that are intended to support instructors during courses and to help students in learning correct technique. Our approach is especially useful when the spatial separation between the instructor and the student is too great to talk or when the instructor cannot focus all of his attention on an individual student. To demonstrate the feasibility of such systems and their potential during training, we have iteratively designed and tested a wearable system for snowboard training, in collaboration with snowboarding instructors and students. This system classifies basic context information on the slope and can be used to detect and to respond to common snowboarding mistakes in realtime. Tiny sensors, unnoticed by humans when woven into clothes or attached to sports equipment, measure body movements and posture during training. Actuators, such as vibration motors, render tactile patterns across the body that alert users to incorrect movements and that communicate hints for corrections. Audio messages through headphones are an alternative way to provide instructions, however, they are less appropriate during the ride. Audio feedback hides valuable environmental cues, such as sound coming from approaching skiers or sound stemming from changing snow conditions.

Another promising application domain for the described feedback systems is daily physical activity. For example, to correctly pick up a heavy box from the floor, the upper body should remain straight while bending and stretching the legs. Many people, however, keep their legs straight and bend only their upper body downwards from the waist to 
lift the object. These movements can lead to serious back injuries when executed over and over again. A wearable systems that monitors body movements can alert people to such wrong habits and signal how to adjust posture.

\section{CONTRIBUTIONS}

This work outlines the rationale for wearable automatic sports assistants and provides an example of such a system in a real-world setting. We start by introducing our vision of future sports training and the benefits that automatic feedback systems offer to both instructors and students. We then describe the hardware design of our system, which was motivated by interviews with snowboarding instructors. Examples and results from a field study conducted on the slope illustrate that our approach can be used to develop an interactive system for supervising posture and for teaching correct snowboarding technique. Results from this work can be applied to other domains where physical activity and the correct execution of movements is essential.

\section{RELATED WORK}

The growing interest in wearable computing for recognizing and correcting physical movements manifests in a variety of projects. Michahelles et al. [7] developed a system to collect data of a skier's run for off-line analysis. Similar to SESAME ${ }^{1}$, this system targets coaches and elite athletes who aim at improving sports performance. Takahata et al. [12] presented a realtime learning environment that uses audio feedback to teach the correct timing of a single karate punch. Kwon et al. [4] developed a motion training system for taekwondo. Kunze et al. [3] conducted an experiment to recognize tai chi movements. Chi et al. [1] built a wearable sensing system to support judges in scoring taekwondo sparring matches. Lindeman et al. [5] described a setup to assist patients during physical therapeutical exercises. Other systems were designed for leisure and fun. Müller et al. [8], for example, presented an exertion interface that supports sports over a distance and fosters social interaction.

Some of these systems, however, were not designed for realtime data analysis and feedback [3, 7]. Existing interactive systems recognize only simple movements, such as punches $[1,8,12]$. Other approaches require expensive hardware to capture body motions, use powerful processors to analyze sensor data, and rely on prior training on a large user database to build appropriate models that recognize and classify motions [3, 4]. Another disadvantage most systems face is their inherent design, which limits their usage to the lab. Only few systems have been designed to work in mobile or outdoor settings [7].

\section{THE VISION OF SPORTS TRAINING}

To illustrate benefits that automatic feedback systems could offer during training, we have chosen the domain of winter sports. The distance on the slope prohibits immediate communication between instructor and students. Thick ski suits conceal body movements and make it difficult to assess the performance of a student who rides far away. Moreover, the trainer has to split his attention across several students who participate in the course, which further reduces the frequency of feedback that an individual student receives.

\footnotetext{
${ }^{1}$ Sensing for Sport And Managed Exercise, sesame.ucl.ac.uk
}

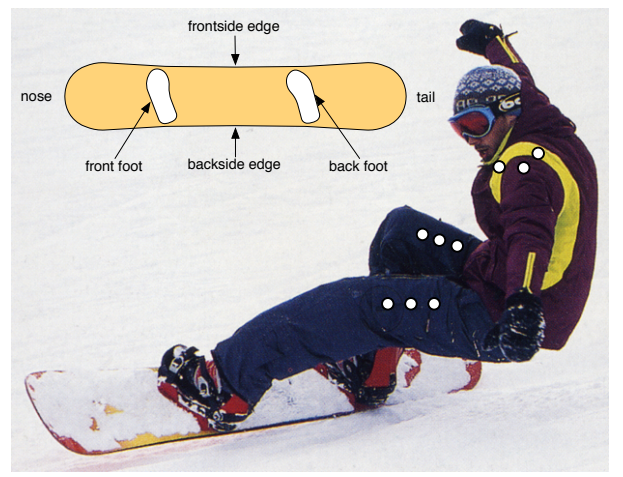

Figure 2: Riding with too much weight on the back foot can lead to falls (photo by Martin Schliephake [2]). Future training systems could use actuators on the body (indicated by white dots) to provide tactile instructions for corrections. The upper sketch illustrates snowboarding terminology.

Snowboarding instructors could use automatic feedback devices as tools that supervise and correct students' mistakes during exercises. A trainer might want to focus on a particular mistake that she noticed while observing one of her course participants. For example, a student might incorrectly shift his weight too much towards the back foot during turns (Fig. 2). To increase the student's awareness of his wrong movements and to support him in correcting weight distribution during the ride, as opposed to providing suggestions for improvement only after the ride, the wearable assistant takes over the task of analyzing posture using on-body sensors. The system further provides immediate feedback to alert the rider to incorrect postures. If an incorrect weight distribution during turns is detected, a unique tactile pattern will be rendered at the student's left thigh (the front foot) to indicate correct weight distribution on the snowboard. This tactile feedback, made of a sequence of short vibrations running downwards on the thigh, signals shift your weight from the back foot towards the front foot.

Once the student has learned to better adjust his weight distribution on the board, the instructor might decide to focus on another mistake, such as counter-rotation during turns. For this, the wearable training system measures the orientation of the upper body relative to the orientation of the snowboard. If an incorrect upper body rotation is detected, a unique tactile pattern will be rendered around the student's right shoulder to signal that the upper body should rotate towards the right instead of towards the left (Fig. 3).

Automatic training systems also offer benefits to amateur and experienced students who ride alone. Even skilled riders may have difficulty maintaining proper technique on challenging slopes. Moreover, a rider's subjective perception of his body posture often deviates from his actual stance. The wearable assistant continuously monitors the rider's performance during descents and provides immediate feedback how to correct posture and fine-tune movements.

\section{STUDY OVERVIEW}

We started by conducting an exploratory formative study with domain experts to better understand the problem and to inform our design of a wearable prototype. Following 


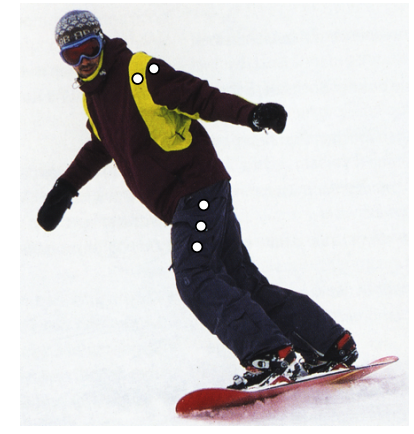

Figure 3: The student rotates his upper body to the left, which is against the current riding direction towards the right. This counter-rotation makes it difficult to introduce the next turn towards the left (photo by Martin Schliephake [2]). The white dots illustrate the possible placement of actuators.

our interviews with instructors, an initial study in the field helped us to validate the concept, to experiment with different sensors, and to iteratively test and improve our system. Finally, we conducted an extensive user study on the slope with potential end-users. During this study, we collected and analyzed sensor data in order to investigate whether it was possible to classify basic context information that is required to further interpret body movements, to recognize mistakes, and to teach proper technique in realtime.

\section{INFORMING THE DESIGN OF SNOW- BOARD TRAINING SYSTEMS}

\section{Interviews with Instructors}

We interviewed six snowboarding instructors to better understand how they teach and to gain more insight into the most common mistakes that occur in this sport. The instructors confirmed our initial assumptions that they cannot observe all students at the same time on the slope. They explained that it is often impossible to give students instant feedback during an exercise. A student receives immediate feedback only in cases when the instructor slowly rides beside a beginner during the first lessons.

All of the interviewed instructors stated that our idea to provide instant feedback during training could be useful to support both instructors and students during courses. They imagined using a wearable assistant themselves to fine-tune their own movements as well as using the system in their own courses, allowing advanced students to focus on a particular mistake. The instructors also pointed out that many beginners do not perceive or do not react to immediate feedback while they perform an exercise. Beginners are usually too focused on keeping their balance. This suggested that our system might be more useful for students who already have some experience in snowboarding, such as advanced and experienced riders who want to improve their skills.

\section{Snowboarding Mistakes}

Three common snowboarding mistakes [2] were identified during the interviews: Incorrect weight distribution during turns, incorrect rotation of the upper body during turns, and insufficient knee bending. The neutral position denotes the correct pose of a rider on the snowboard and can be used as reference posture to find potential mistakes during the ride. In neutral position, the weight is central over the board and distributed equally between both feet. Legs and ankles are flexed. This flexing acts as a natural suspension to compensate uneven terrain, for example when riding over bumps in the slope. Shoulders and hips are in line with the feet's stance on the snowboard. The head is up and the rider looks towards the riding direction. We will now briefly summarize the three identified mistakes and mention possible sensors that can be used for automatically analyzing posture and detecting erroneous body movements.

Incorrect weight distribution (Fig. 2): To begin a new turn, the weight should be shifted towards the front foot. After pivoting the board, the rider should distribute his weight equally between both feet. However, many riders tend to incorrectly lean their upper body towards the tail. The resulting posture shifts the weight towards the back foot, which makes it difficult to initiate and to pivot the snowboard across the fall line. This mistake can be addressed with force-sensitive resistors (FSR) inserted into the boots, which measure the weight distribution between both feet.

Incorrect upper body rotation (Fig. 3): The rider's shoulders and the waist should remain in the same plane with the feet's stance during the ride. To introduce a turn, the upper body should be rotated towards the intended riding direction. Yet many riders tend to twist their upper body contrary to the turning direction, which makes it hard to initiate and perform the next turn. This mistake can be addressed with gyroscopes or with digital compasses that measure the orientation of the upper body relative to the orientation of the snowboard. Stretch sensors woven into the garment [6] or magnetic field sensors [9] might be alternative approaches for identifying this particular mistake.

Insufficient knee bending: Bending the legs helps as a natural suspension to compensate for uneven terrain. Some riding techniques also require alternating from high to low (flexing and extending the legs) while pivoting the board during turns. Many riders, however, cannot correctly assess if they bend their legs sufficiently or not. They tend to stretch their legs and to bend their upper body downwards from the waist. This mistake can be addressed with bend sensors or with stretch sensors attached to the rider's joints, which measure the degree of flexion.

\section{System Setup}

The conducted interviews with domain experts helped us to better understand the requirements for a snowboard training system that senses motion, detects mistakes, and provides feedback in realtime. We were first looking at existing hardware solutions that we could use for building a wearable prototype. In particular, we were interested in a system that allowed us to easily experiment and exchange different sensors and actuators on the fly and to log sensor data for initial tests. Moreover, the system should allow us to implement customized algorithms for context and mistake recognition as well as higher application logic for comparing the student's current posture to the intended riding posture required by particular snowboarding techniques. Although specialized sensor systems for motion capture exist (see xsense.com), these systems did not prove to be convenient for our tasks. Such systems are still limited in terms of customizability and programmability. We thus decided to 


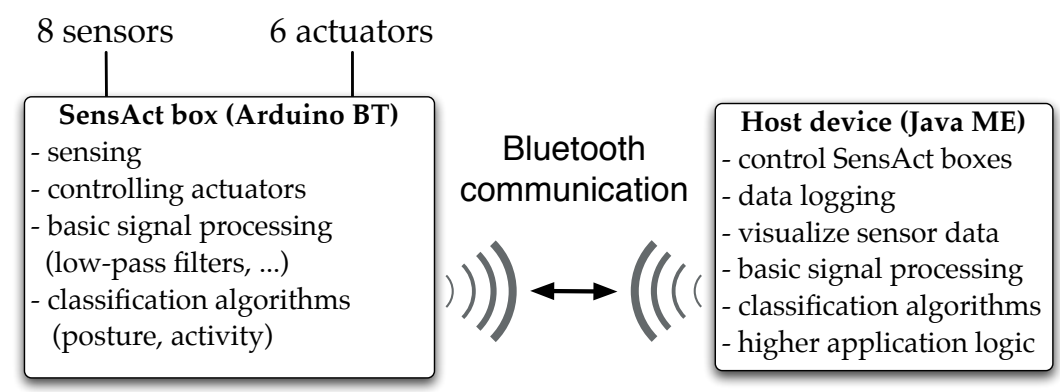

Figure 4: The system architecture of our platform.

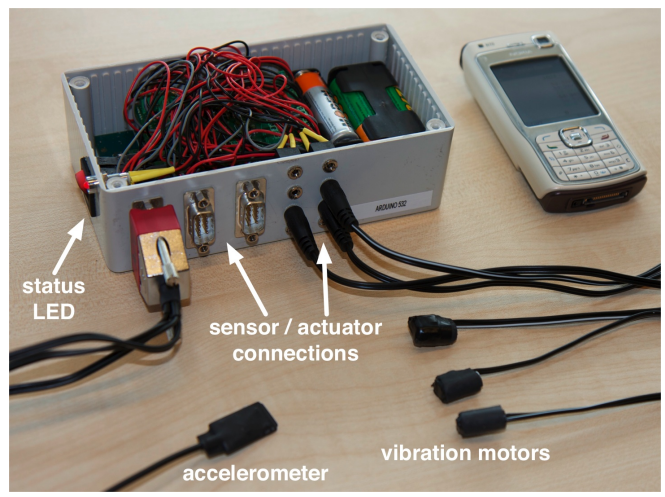

Figure 5: The SensAct box allows replacement of sensors and actuators at runtime.

design our own sensor/actuator box that better meets the requirements detailed in our vision. Throughout the conducted user studies described in this work, we continued to iteratively improve and refine our platform for outdoor use.

\section{Hardware Platform}

Our system consists of two units: A standard mobile phone and our custom-built SensAct sensor/actuator box. The mobile phone, a Nokia N70 in our case, acts as host device. Fig. 4 illustrates the system architecture and the tasks of each unit. The SensAct box (Fig. 5) contains a Bluetooth Arduino board ${ }^{2}$, an open-source electronics prototyping platform. A custom-built motor controller for the Arduino provides vibrotactile feedback through actuators, such as small vibration motors. Actuators are connected via TS, sensors via SUB-D connectors to avoid loose connections. The dimensions of the SensAct box are $15 \times 8 \times 5 \mathrm{~cm}(6 \times 3 \times 2 \mathrm{in})$.

\section{Software Architecture}

Bluetooth allows the host device to concurrently control up to seven SensAct boxes. We developed software libraries for both the SensAct box (programmable in a C-like language) and the host device (Java, Python). These libraries include algorithms for basic signal processing, posture classification, and activity recognition (described below) used for implementing stand-alone programs. Programs can be freely distributed between several SensAct boxes. All algorithms can alternatively run on the host, using raw sensor measurements streamed in realtime from multiple SensAct boxes.

\footnotetext{
${ }^{2}$ www.arduino.cc
}

The host sends control messages to the SensAct boxes. These messages define, for example, the sampling rate for sensors or trigger actuators for rendering vibrotactile feedback. Other control messages start and stop the streaming of raw sensor data or of classification results. We found no noticeable delay caused by the Bluetooth communication between SensAct box and host device. For example, the average time to send a six bytes command that activates actuators connected to the box is approximately $39 \mathrm{~ms}$.

\section{Initial Testing of the Technology}

Using our sensor system, we conducted an exploratory pilot study in an indoor winter sport resort with three snowboarders at advanced beginner level. This study aimed at testing our system under real-world conditions. We further collected a first set of raw sensor data for off-line analysis.

Insoles with TouchMicro- $10^{3}$ FSRs were inserted into the boots to measure the amount of force applied by the feet. We placed two FSRs under the ball of each foot (1st and 5th metatarsal bones) and one FSR under the heel (calcaneus). These sensors had a diameter of $10 \mathrm{~mm}$ and measured forces up to $667 \mathrm{~N}(68 \mathrm{~kg})$. To measure the amount of knee flexion, BendShort $^{3}$ bend sensors ( $87 \mathrm{~mm}$ long) were wrapped in foam to increase their robustness and attached to the back of each knee with kneepads. Though some participants stated they noticed sensor cables under the insoles and the foam at the knees, this equipment did not cause discomfort and did not hinder them in their movements. However, adjusting all sensors properly was time consuming and lasted one hour.

All six sensors were connected to our custom-built sensor box. We further used two Shake SK6 ${ }^{4}$ devices to measure rotation of the rider's upper body relative to the rotation of the snowboard. The Shake SK6 is a matchbox-sized inertial measurement unit with built-in digital compass algorithm and Bluetooth communication. We attached one Shake device to the lower front leg and another Shake device to the upper body of the rider with hook and loop fasteners.

Fig. 6 shows our prototype system with all sensors used for data collection during the field study. The host device connected to all three sensor packs over the Bluetooth serial port profile and recorded raw sensor data at $20 \mathrm{~Hz}$. We additionally captured the subjects on video while descending. The distance between the starting point on the slope and the camera was about 60 meters. We chose this distance to simulate a setting that was similar to what snowboard instructors typically observe during courses. Each subject

\footnotetext{
${ }^{3}$ www.infusionsystems.com

${ }^{4}$ www.samh-engineering.com
} 


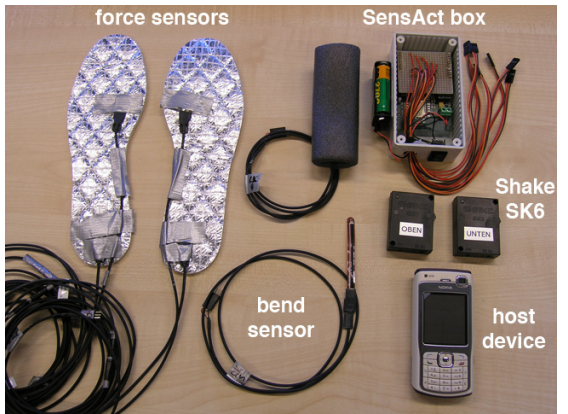

Figure 6: The first hardware prototype and sensor setup to measure the rider's posture and motions.

descended the same part of the slope two times. All participants wore the SensAct box in a small waist bag and the host device in their pocket. We experienced no data loss or connection problem and found no significant overhead in the Bluetooth communication between the wireless devices.

\section{Preliminary Results and Instructors' Opinion}

To inspect the raw sensor measurements off-line, we synchronized sensor and video recordings using custom-written software. The sensor recordings revealed that setting thresholds on sensor signals was sufficient to reliably detect the amount of knee bending and to estimate the weight distribution between toes and heels on the snowboard. Acceleration during the ride, however, influenced the compass measurements, thus making it difficult to reliably detect incorrect rotation of the upper body only with a digital compass.

Following our initial tests, we presented our wearable system to eleven snowboard instructors and 28 skiing instructors who participated in an advanced training course for instructors. One of the snowboarders and three of the skiers were professional instructors who led the course. We introduced the participants to our vision of a wearable snowboarding assistant and explained how the sensing system worked. Finally, we visualized sensor data collected during initial testing. Examples included amount of knee bending and weight distribution between toes and heels, since these sensors provided the best measurements during descents.

$73 \%$ of the snowboard instructors and $68 \%$ of the ski instructors considered our idea to sense the rider's motion and to provide realtime feedback during snowboard and ski training to be potentially very valuable. The other participants questioned whether an automatic system could correctly recognize mistakes during the ride. They argued that without detailed knowledge about the slope it is impossible to distinguish between correct and incorrect movements. For example, in some cases, an instructor needs to see the gradient of the slope in order to assess if a student bends his legs sufficiently. Moreover, classifying certain body movements as correct or wrong also depends on the current context during the descent. For mistake recognition to work, the system needs to distinguish whether the rider performs a frontside turn or a backside turn, which determines the set of valid movements. While the first objection is valid for a subset of all possible mistakes, our prototype system might still be used to detect an incorrect posture or movements that are independent of the slope's characteristics, such as wrong weight distribution or upper body rotation during turns.

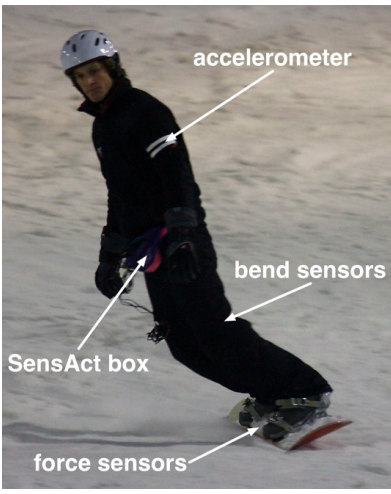

Figure 7: A user with the prototype system.

\section{SYSTEM EVALUATION WITH USERS}

Motivated by the results of our initial test and the instructors' opinion, we decided to conduct a formal user study to further analyze sensor measurements that proved most promising for building a wearable snowboarding assistant at the present time. The main goal of this study was to investigate if it was possible to detect the instant when a rider began a new turn and to determine if the turn was performed on the frontside edge or on the backside edge. During frontside turns the snowboarder faces uphill and rides on the frontside edge, during backside turns he faces downhill and rides on the backside edge (see sketch in Fig. 2). As described by instructors, this context information during descents is essential to automate the task of recognizing mistakes that do not conform to correct riding techniques. We additionally tested algorithms to recognize incorrect weight distribution and insufficient knee bending. Finally, we evaluated an algorithm for activity recognition that classifies whether participants were pausing or riding. Algorithms have been evaluated off-line on raw measurements recorded on the slope. We performed this step to optimize algorithms for the best recognition accuracy across all participants, before the final implementation on our wearable platform.

\section{Experimental Setup}

Eight snowboarders (one female) aged 23-27 participated in this study. On a scale ranging from level one (beginner) to level five (expert), one participant rated his skills as level one, two as level two, three as level three, and two as level five. One of the expert snowboarders was a snowboard instructor. Four participants stated that they had attended a training course to improve their skills in snowboarding. On average the subjects snowboarded between one and two weeks per year during holidays.

We used a similar hardware setup as in the pilot study to record raw sensor data and to simultaneously capture our participants on video. Sensor measurements comprised data from six force sensors inserted into the boots, two bend sensors attached to the knee, and one $2 \mathrm{D}$ accelerometer at the upper arm (Fig. 7). The SensAct box sampled data at $50 \mathrm{~Hz}$ and streamed measurements to the host device.

The distance between the starting point on the slope and the camera was about 140 meters. By choosing this distance, the subjects gained higher speed and performed more consecutive turns as compared to the pilot study. For each subject we recorded several runs. For some runs, partici- 
pants were instructed to descend the slope as they always did. For other runs, participants were introduced to an alternative snowboarding technique that required stretching and flexing the legs during turns. We asked participants to ride according to this alternative technique in order to verify whether they can perform the task as required and whether sensor measurements revealed knee flexion during turns.

\section{Turn and Edge Detection}

Knowing the riding edge and the instant when a new turn begins is essential to further interpret if a rider's movements are correct or wrong. To determine whether the frontside edge or the backside edge is subject to the highest pressure, we compare FSR measurements to reference values recorded during an initial calibration step while standing on level ground. This comparison yields whether the rider performs a turn on the frontside edge or on the backside edge. Transitions from one edge to the other edge correspond to the beginning of turns while pivoting the board.

The algorithm works as follows: Mean shifting first adjusts raw sensor values by subtracting the corresponding reference values. As a result, the new reference values become 0 for all sensors. Simple exponential smoothing reduces sensor noise. The algorithm then sums the forces measured under the balls of feet $(S B)$, the forces measured under the heels $(S H)$, and computes the difference $D=S B-S H$. Simple moving average with window size $w$ returns the mean weight distribution $E=S M A_{w}(D)$ on the snowboard. $E$ yields whether the weight is towards the balls or towards the heels and determines the riding edge. A threshold value $T_{E}$ defines the tolerance range around the reference value 0 , where we regard the weight distribution between the balls and the heels as evenly distributed. The rider is riding on the frontside edge if the weight distribution is towards the balls $\left(E>T_{E}\right)$. The rider is riding on the backside edge if the weight distribution is towards the heels $\left(E<-T_{E}\right)$. The rider pivots the board from one edge to the other edge if the weight is neither too much towards the balls nor too much towards the heels $\left(-T_{E} \leqslant E \leqslant T_{E}\right)$.

To determine the best recognition rate depending on different exponential smoothing factors $\alpha(0 \leqslant \alpha<1)$, we compared the output of our algorithm to the turns on the video footage. We considered true positives (recognized turns), false negatives (missed turns), and false positives (false alarms). Experimental sessions using the recordings from our pilot study allowed us to choose a threshold value $T_{E}=50$ and a window size $w=25$ (roughly $500 \mathrm{~ms}$ ) to compute $E$. We used the first runs from our subjects as training set and the last runs as test set to evaluate the described algorithm for turn and edge detection.

\section{Results}

Fig. 8 shows an example output of the algorithm (black continuous line) for turn and edge detection applied to the raw sensor measurements recorded from two FSR sensors per foot (1st metatarsal bone, calcaneus). Classification results for the training set were independent of the smoothing factor $\alpha$. All 56 turns were correctly classified either as frontside turns or as backside turns. For the test set, we found an exponential smoothing factor of $\alpha=0.9$ to rank highest. The algorithm correctly classified 59 out of 61 turns $(96.7 \%)$ and reported two temporary false positives. Lower smoothing factors ranked only slightly lower with at

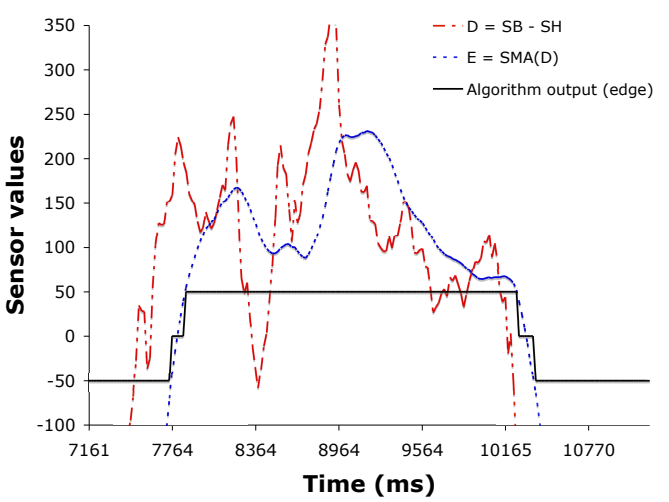

Figure 8: Output of the algorithm for turn and edge detection (black continuous line): Backside edge $(Y=-50)$, frontside edge $(Y=50)$, pivoting $(Y=0)$. Transitions between -50 and 50 indicate the beginning of new turns.

most five temporary false positives for the test set. Using all three FSR sensors instead of two FSRs per foot did not significantly improve classification results.

\section{Discussion}

The high recognition accuracy shows that turns and the riding edge can be accurately classified with only two force sensors per foot. The slightly lower accuracy of the algorithm to recognize turns in the test set might stem from the single calibration step that we performed for each subject only before the first descent. FSR sensors that were not appropriately placed under the feet can further skew measurements and influence results; for some subjects we experienced difficulties when trying to fit the insoles with the sensors into the snowboard boots. Sensor cables under the soles of the feet and displaced sensors after consecutive descents might be other reasons. Repeating the calibration step before each descent and using boots with built-in sensors might help to improve the overall recognition accuracy in future.

The threshold $T_{E}$ corresponds to the sensitivity of the algorithm to detect the riding edge; the algorithm interprets a shift in weight towards the toes (heels) as riding on the frontside (backside) edge. Low thresholds can be used to detect turns and to identify the riding edge for beginners who do not yet have the skills to increase the edge angle of the board and thus rather slide down the slope. For expert riders, who are skilled enough to carve on the edges, higher thresholds can assess the quality of their carving technique.

A similar algorithm as used for turn and edge detection can theoretically estimate the weight distribution between the front foot and the back foot. Evaluation revealed that estimating the weight between both feet on the snowboard, as opposed to between toes and heels, was not accurately possible even with three FSR sensors inserted into each boot. The front binding, which fastens the front part of the foot to the board, influenced measurements and did not yield reliable results. Future work should investigate whether more FSR sensors per foot, sensors built directly into the board or into the binding might help to detect whether riders incorrectly shift their weight towards the back foot during turns. 


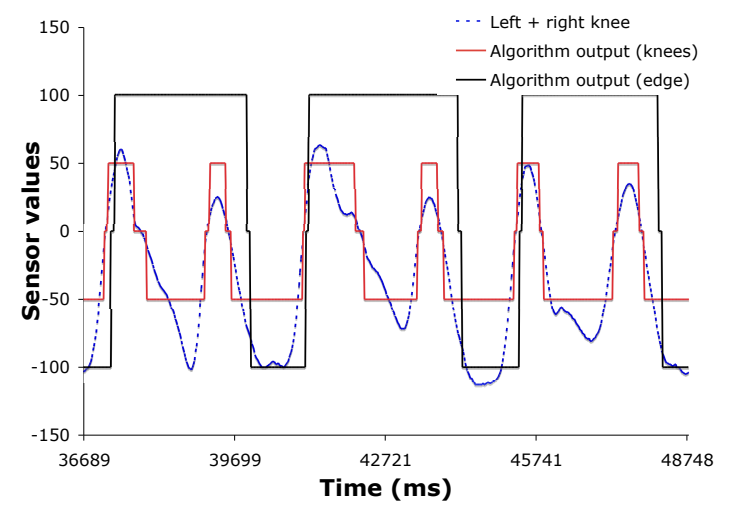

Figure 9: Amount of knee flexion during the ride (red line): Flexed legs $(Y=-50)$, stretched legs $(Y=50)$. Riding edge (black line): Backside edge $(Y=-100)$, frontside edge $(Y=100)$.

\section{Knee Flexion during the Ride}

The two bend sensors attached to the back of both knees measured knee flexion and duration of flexion during the ride. This information revealed whether participants rode with straight knees for extended time periods. For one of their descents, the subjects were instructed to alternate between extending the legs while pivoting the board and flexing the legs during traversal. To assess whether the participants performed this technique as required, we compared bend sensor measurements to a threshold value $T_{K}$ that we recorded in neutral position before descending the slope. Fig. 9 depicts knee flexion in relation to the riding edge for one participant; the student stretched and bent her legs sufficiently while pivoting the snowboard. The temporal component of the classification results further helped to assess the quality of the student's technique. Flexion almost coincided with pivoting from the backside edge to the frontside edge but occurred slightly before pivoting from the frontside edge to the backside edge. Though bend sensors worked reliably during most descents, the foam with the sensors occasionally slipped out of the kneepad and had to be readjusted.

\section{Activity Recognition: Stop and Go}

A wearable snowboarding assistant should analyze posture and provide feedback only if the student is riding. During breaks the system should remain idle. To distinguish between riding and standing, we measured acceleration in direction to the descent and vertical to the slope using one $2 \mathrm{D}$ accelerometer, which was attached to the upper arm. Our algorithm summed the standard deviations of both acceleration components over the last 0.5 seconds and compared the result to a reference value measured while at rest. This simple activity recognition algorithm achieved an accuracy of $80.5 \%$ in classifying whether participants were riding or pausing. False positives occurred while riding at low speed, such as during the slow deceleration phase before rest or during the slow acceleration phase after rest. The accuracy of our approach to detect activity is only slightly lower compared to [10], which extracted more features and used a clustering algorithm to differentiate between six activities.

\section{DISCUSSION OF RESULTS}

The described algorithms set thresholds on sensor signals to classify posture and activity. While this straightforward approach does not require training of recognition models in advance, our method does not scale to continuous motion. Machine learning techniques, such as hidden Markov models, better address recognition of such movements [3, 4]. Further work has to be done until all snowboarding mistakes can be accurately identified using body-worn sensors.

Despite that limitation, the obtained results are adequate to build interactive training systems that do not depend on tracking and differentiating fluent and continuous body motions. Classification results presented in this work reveal transitions between movements, such as changing weight distribution or several degrees of joint flexion, and yield a posture model of the body. This model allows to trigger realtime feedback intended to demonstrate correct execution of body movements that conform to specific sports techniques.

State machines can be used to implement such a behavior. In our case, feedback could be triggered at the appropriate time during descents, reminding and guiding students during turns. For example, our system knows when transitions between turns occur and whether the student currently performs a frontside turn or a backside turn. This information can be used to instruct correct technique for basic turns: While riding on the frontside edge, the wearable assistant notifies the student to shift your weight to the front foot, followed by turn your upper body to the left to introduce the next turn. While riding on the backside edge, instructions are shift your weight to the front foot, followed by turn your upper body to the right. To sensitize students for correct timing to alternate from flexing and extending the legs during consecutive turns, the system provides instructions to bend your legs as soon as a new turn has been detected, then to stretch your legs followed by turn your upper body to the left (right) in order to introduce the next turn. The described approach can be compared to an instructor who pushes or pulls the student in the correct directions to indicate correct posture, similar to tennis and golf instructors who guide the students' arms to demonstrate correct strokes.

Tactile motion instructions [11] are vibration patterns triggered across the body, as introduced in our vision of future sports training. These instructions represent specific body movements, such as shift your weight to the left foot, turn your upper body to the right, or bend your legs. We proposed a set of ten such instructions and used our system to conduct a study with ten snowboarders. The task was to identify randomly triggered tactile instructions across the body while descending the slope. Results revealed that our participants perceived very well tactile instructions (87\% correct) as compared to corresponding audio instructions (97\%) played back over earplugs while snowboarding. Moreover, students responded on average one second faster to tactile instructions than to audio instructions. This faster response time to tactile instructions over their audio counterparts is an important advantage for sports such as snowboarding; the rider has to react quickly to changing conditions and continuously adjusts posture to keep balance. A post-test questionnaire revealed no significant difference between audio and tactile instructions regarding comfort of the system, intuitiveness of instructions, or distraction during the ride.

The results presented in this work further demonstrate that wearable computing applications intended for supervis- 
ing and alerting users to wrong movements performed during daily physical activities can be implemented and executed in realtime on standard low-power computing devices. Referring back to the second introductory example, bend sensors woven into trousers and a tilt sensor (or an accelerometer measuring tilt) attached to the upper body are sufficient to monitor whether one correctly bends the legs or whether one incorrectly bends only the upper body to lift an object from the floor. Tactile motion instructions triggered immediately at the torso and at the upper thighs can signal the correct execution of movements to prevent injuries.

\section{SUMMARY AND CONCLUSIONS}

We presented our work on wearable automatic feedback devices for assisting people during daily physical activities and for supporting instructors and students during sports training. These systems are supposed to detect harmful and incorrect posture and movements using body-worn sensors, and to provide tactile feedback for corrections with actuators placed at key positions across the body.

As an example of such systems, we have developed a first interactive assistant for snowboard training. Based on interviews with domain experts, we have designed a hardware platform that classifies basic context information and that can be used to demonstrate and to indicate appropriate timing and correct execution of specific body movements. Context-recognition was evaluated during a study with endusers on the slope. The results show that simple feature extraction derived from sensors and setting thresholds on sensor signals is sufficient to reliably identify the riding edge and to measure knee flexion. These classification results allow to build a posture model of the body and to compare currently performed movements to specific sports techniques. Apart from measuring reference values in neutral position while at rest, no subject specific training for creating recognition models was required. The described algorithms run on standard low-power micro-controller devices in realtime.

According to interviewed snowboarding and skiing instructors, the proposed approach of automatic performance analysis with immediate tactile feedback during exercises has the potential to change current training methods and to enhance the learning experience of winter sport practitioners. The system we described was tailored specifically for snowboard training but can be adapted to other sport domains as well, such as skiing, surfing, freeboarding, riding, or dancing.

\section{FUTURE WORK}

A wearable system for accurately tracking the relative position and orientation of body parts using magnetic field technology has been recently demonstrated [9]. This approach can help to improve the recognition of incorrect upper body rotation while descending the slope. Alternatively, stretch sensors and gyroscopes attached to the rider's torso might help to reliably detect this particular mistake. Similarly, analyzing incorrect weight distribution towards the back foot while descending the slope needs further investigation with different sensor setups and snowboard bindings.

Tactile motion instructions are a promising technique for providing immediate feedback for corrections during sports training. We further plan to use our system to explore the influence of such realtime tactile feedback on the learning experience of snowboarding students during real courses.

\section{ACKNOWLEDGMENTS}

We would like to thank SnowWorld.com for their kind support provided during our user studies. This work was funded in part by the German B-IT Foundation.

\section{REFERENCES}

[1] Chi, E. H., Song, J., and Corbin, G. "Killer App" of Wearable Computing: Wireless Force Sensing Body Protectors for Martial Arts. In 17th Symposium on User Interface Software and Technology, pages 277-285. ACM Press, October 2004.

[2] Dt. Verband f. d. Skilehrwesen, Interski Deutschland. Snowboard-Lehrplan. BLV Buchverlag, München, 2003.

[3] Kunze, K., Barry, M., Heinz, E., Lukowicz, P., Majoe, D., and Gutknecht, J. Towards Recognizing Tai Chi An Initial Experiment Using Wearable Sensors. In 3rd Int. Forum on Applied Wearable Computing. Springer, April 2006.

[4] Kwon, D. Y. and Gross, M. Combining Body Sensors and Visual Sensors for Motion Training. In Int. Conf. on Advances in Computer Entertainment Technology, pages 94-101. ACM Press, New York, 2005.

[5] Lindeman, R.W., Yanagida, Y., Hosaka, K., and Abe, S. The TactaPack: A Wireless Sensor/Actuator Package for Physical Therapy Applications. In Virtual Reality, pages 337-341. IEEE, Washington, March 2006.

[6] Mattmann, C., Amft, O., Harms, H., Troster, G., and Clemens, F. Recognizing Upper Body Postures using Textile Strain Sensors. In 11th Int. Symposium on Wearable Computers, pp. 1-8. IEEE, 2007.

[7] Michahelles, F., Crameri, E., and Schiele, B. Design, Implementation and Testing of a Wearable Sensing System for Professional Downhill Skiing in Cooperation with Trainers. In 2nd Int. Forum on Applied Wearable Computing. Springer, March 2005.

[8] Müller, F., Agamanolis, S., and Picard, R. Exertion Interfaces: Sports over a Distance for Social Bonding and Fun. In Int. Conference on Human Factors in Computing Systems, pages 561-568. ACM Press, New York, April 2003.

[9] Pirkl, G., Stockinger, K., Kunze, K., and Lukowicz, P. Adapting Magnetic Resonant Coupling Based Relative Positioning Technology for Wearable Activitiy Recogniton. In 12th Int. Symposium on Wearable Computers, pages 47-54. IEEE, Piscataway, Sept. 2008.

[10] Randell, C. and Müller, H. Context Awareness by Analysing Accelerometer Data. In 4th Int. Symposium on Wearable Computers, pages 175-176. IEEE Press, Washington, 2004.

[11] Spelmezan, D., Jacobs, M., Hilgers, A., and Borchers, J. Tactile Motion Instructions for Physical Activities. In Int. Conference on Human Factors in Computing Systems. ACM Press, New York, April 2009.

[12] Takahata, M., Shiraki, K., Sakane, Y., and Takebayashi, Y. Sound Feedback for Powerful Karate Training. In Int. Conference on New Interfaces for Musical Expression, pages 13-18. National University of Singapore, Singapore, 2004.

[13] Wulf, G. Attention and Motor Skill Learning. Human Kinetics, 2007. 